\title{
Influence of injection conditions on viscoelastic ligament dynamics
}

\author{
Christophe Dumouchel*, Marie-Charlotte Renoult, Christophe Tirel, Louise Cottier \\ ${ }^{1}$ CNRS UMR 6614 CORIA, Normandy Univ., UNIROUEN, INSA Rouen, 76000 Rouen, France \\ *Corresponding author email: Christophe.Dumouchel@coria.fr
}

\begin{abstract}
This paper presents an experimental work on the determination of viscoelastic solution properties from the analysis of their behavior when subject to the capillary instability. Known as the jet rheometer method, such an approach is reserved for low-viscous viscoelastic solutions for which commercial techniques are unsuitable. In the present work, the injection conditions are free and cover a wide range of operating points. The analysis is statistical in nature and uses a multi-scale description tool that allows access to all dynamics involved and, in particular, to those of small characteristic scales. The results are numerous and evidence the presence of polymer mechanical degradation due to the injection. The influence of the operating conditions on this mechanism is highlighted.
\end{abstract}

\section{Keywords}

Dilute polymer solution, ligament behavior, extensional properties.

\section{Introduction}

Filament-stretching rheometers are devices for measuring the relaxation time $t_{r}$ and the extensional viscosity $\eta_{E, a p p}$ of viscoelastic liquids by following the capillary thinning of a filament stretched between two plates ([9,7] among others). These devices are not appropriate for low-viscosity viscoelastic liquids such as very dilute polymer solutions. The recommended alternative in such cases is to use a technique based on jet experiments and referred to as the jet rheometer in the literature $[8,5,6]$. This technique consists in following the decrease of the diameter of the ligament persisting between two consecutive growing bulges. Both the relaxation time and the extensional viscosity can be measured from this dynamics.

This paper presents experimental results obtained with a jet rheometer whose protocol has been established during the last four years [12]. This protocol differs from those of the literature by two main aspects. First, most jet rheometers impose both the amplitude and frequency of the initial perturbation of the jet. This is not the case in the present protocol that considers free liquid jet experiments. Consequently, a statistical analysis is performed and the protocol reports averaged relaxation time and terminal extensional viscosity. Second, in the literature, the image analyzing step is meant to access to a measurement of the ligament diameter as a function of the distance from the injector. This of course requires a prior detection of the ligament. (To this respect, the use of a controlled initial perturbation eases this detection if the jet is enlightened at an appropriate frequency, which is usually done.) In the present jet rheometer, this step is not needed. The image analysis leads to the measurement of the scale distribution of portions of the jet. This distribution provides a geometrical, morphological and topological description of the jet. The dynamics of small characteristic scales reported by the temporal evolution of this distribution is the same as the one of the ligament diameter. The relaxation time and the terminal extensional viscosity of the viscoelastic solution can be measured from this dynamics as reported in the literature [12].

In this paper, a wide range of experimental results is presented. They illustrate the influence of the jet initial diameter and velocity and of the polymer concentration and molar weight. Among other observations, a correlation between the average relaxation times and terminal extensional viscosities is reported. It appears to align with the experimental results and correlation we owe to Stelter et al. $[10,11]$. 


\section{Experimental method, analysis and measurements}

The experimental set-up, the image analysis and the measurements are described in details in [12] and are summarized here only.

\section{Experimental method}

The experimental set-up produces free falling cylindrical jets using injectors whose nozzle is made of a disc perforated with a circular orifice. The injector is fed by a syringe pump that imposes the flow rate. The optical visualization system is a back-light imaging assembly. It comprises a CAVITAR laser source, a long distance microscope and a camera whose sensor has 29 or 47 Mpixels according to the operating conditions. The duration of the light pulse is chosen equal to $20 \mathrm{~ns}$ to freeze the jet on the image. The image spatial resolution is chosen so that the diameter of the cylindrical jet in its undisturbed part is displayed over at least 80 pixels. This precaution is recommended to perform a multi-scale description of the jet. For the chosen image resolutions, the field of view is not large enough to visualize the jet entirely from the nozzle to the breakup region. The injector is positioned on a 3D displacement system allowing to focus the jet and to select the part of the jet to be visualized. For each operation condition, series of 150 to 300 images of the jet are taken from the injector nozzle exit down to the breakup region.

The viscoelastic fluids are dilute polymer solutions at different concentrations. The experiments were performed with Poly(ethylene oxide) PEO with an average molar mass $M_{w}$ equal to 0.6, 1,5 and $810^{3} \mathrm{~kg} / \mathrm{mol}$. The polymer is dissolved into demineralized water with $5 \%$ in mass of isoproyl alcohol in order to ease the polymer dispersion. The polymer concentration was varied from 5 to $20 \mathrm{ppm}$ in mass of PEO. (Throughout this paper, the concentration information refers to mass proportion.) For each solution, the density, shear viscosity and surface tension are measured at $20^{\circ} \mathrm{C}\left(989 \mathrm{~kg} / \mathrm{m}^{3} ; 1.34 \mathrm{mPa} . \mathrm{s} ; 48.9 \mathrm{mN} / \mathrm{m}\right)$. Seven injector nozzles were used. Their geometric dimensions are gathered in Table 1 as well as the image spatial resolution selected for each of them. The volume flow rate $Q_{v}$ ranges from 1.8 to $5010^{-8} \mathrm{~m}^{3} / \mathrm{s}$.

An example of reconstructed jet is presented in Fig. 1 for which probing eight vertical positions was necessary to visualize the entire jet, i.e., from the nozzle down to the breakup region. The jet evolution shows three stages. Between 0 and about $20 \mathrm{~mm}$ no marked deformation is observed. Between 20 and $25 \mathrm{~mm}$, a sinusoidal deformation develops. Its amplitude increases with the distance from the injector. Between $25 \mathrm{~mm}$ and $44 \mathrm{~mm}$, the jet shows the Bead On A String (BOAS) pattern, characteristic of the capillary instability of viscoelastic jets. Beyond this position, jet breakup occurs.

Table 1. Nozzle diameter $D_{\text {or }}$ and length $L_{o r}(\mu \mathrm{m})$, Image spatial resolution $I R(\mathrm{px} / \mathrm{mm})$ and Analyzing window height $H_{w}(\mathrm{~mm})$

\begin{tabular}{c|ccc|ccc|c}
\hline Ref & B1 & B2 & B3 & B4 & B5 & B6 & B7 \\
\hline$D_{o r}$ & 42 & 105 & 200 & 367 & 480 & 500 & 500 \\
$L_{o r}$ & 300 & 300 & 300 & 1000 & 1000 & 1000 & 5000 \\
$I R$ & 1780 & 1780 & 792 & 600 & 600 & 600 & 600 \\
$H_{w}$ & 1 & 0.5 & 0.5 & 3 & 3 & 3 & 3 \\
\hline
\end{tabular}

\section{Image analysis and measurements}

The aim of the image analysis is to measure the average scale distribution $e_{3}(d, t)$ of the jet as a function of the distance from the orifice, i.e., as a function of time. Introduced in several previous publications [2,12], the scale distribution is the scale derivative of the volume scale distribution which reads as the volume comprised between the liquid-gas interface and its parallel surface at a distance $d / 2$ towards the liquid region, divided by the total initial volume. This parallel surface delimits the so-called $d$-scale eroded system resulting from the erosion at scale $d$ of the initial liquid system. It can be demonstrated that, whatever the shape of the system and 


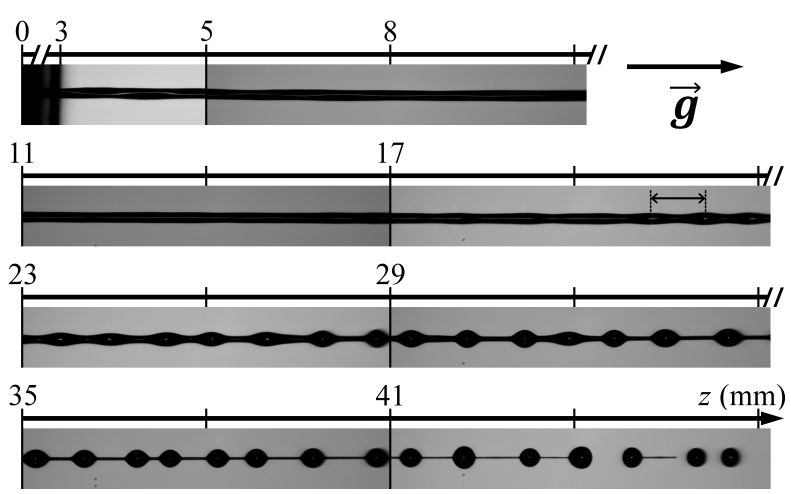

Figure 1. Reconstructed viscoelastic liquid jet.

the value of the erosion scale, the scale distribution is always equal to the surface area of the $d$-scale eroded system divided by twice the total initial volume [3].

To proceed to the measurement of the scale distribution, the images undergo a succession of treatments in order to produce binarized images on which the jet appears in black on a white background. The Euclidean Distance Map (EDM) of the liquid system is then calculated: this step attributes to each liquid pixel a gray level value equals to its distance from the closest interface point. The volume scale distribution and its scale derivative, the scale distribution, are easily calculated on EDM images. (Although images provide a 2D information, the 3D scale distribution is deduced by assuming the axisymmetry of the liquid jets, which seems acceptable considering the axisymmetric nature of the capillary instability mechanism.)

In the present work, this calculation is performed on a small portion of the jet, delimited by a rectangular analyzing window centered at position $z$ (see Fig. 1). The analyzing window height $H_{w}$, whose values are given in Table 1 , is of the order of the naturally selected perturbation wavelength. The local scale distribution is measured on a high number of images (between 100 and 300) and a mean scale distribution $e_{3}(d, z)$ is calculated as the volume-weighted average scale distributions. Then, the spatial $z$ dependence is transformed as a temporal $t$ dependence from the hourly equation of a free-falling object with a velocity $V_{j e t}$. The equivalent time $t$ is defined by:

$$
t=\frac{\sqrt{V_{j e t}^{2}+2 g z}-V_{j e t}}{g}
$$

The mean scale distributions $e_{3}(d, z)$ are then analyzed to identify a specific scale which follows the same time evolution as the diameter of the ligament of the BOAS pattern. It has been theoretically demonstrated [12] that the small scale (called $d_{4}$ at which the distribution $\dot{e}_{3}^{\prime}(d, t)$ peaks (where the prime indicates a scale derivation and the dot, a temporal derivation) has the same dynamics as the ligament diameter. An example of this distribution is plotted in Fig. 2. The scale $d_{4}(t)$ identified in Fig. 2 decreases with time as illustrated in Figure 3.

Works in the literature $([1,7]$ among many others) demonstrate that the ligaments of the BOAS pattern follow two regimes of diameter decrease known as, per order of appearance, the elastocapillary regime and the visco-capillary regime. The elasto-capillary regime takes place when the capillary contraction of the ligament is controlled by elastic effects generated by the polymer unfolding process. This regime is characterized by an exponential temporal decrease of the ligament diameter with a constant deformation rate $\dot{\varepsilon}(t)$. It has been demonstrated [12] that the deformation rate and the apparent elongational viscosity $\eta_{E, a p p}(t)$ are related to the scale $d_{4}(t)$ by:

$$
\dot{\varepsilon}(t)=\frac{-2 \dot{D}(t)}{D(t)}=\frac{-2 \dot{d}_{4}(t)}{d_{4}(t)}
$$




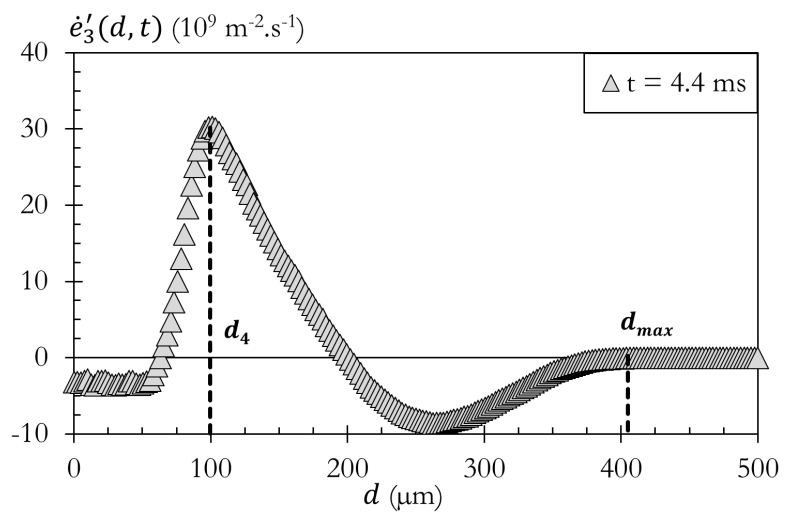

Figure 2. Scale distribution $\dot{e}_{3}^{\prime}(d, t)$ and $d_{4}(t)$ definition (5ppm, B3, $\left.Q_{v}=23.710^{-8} \mathrm{~m}^{3} / s\right)$

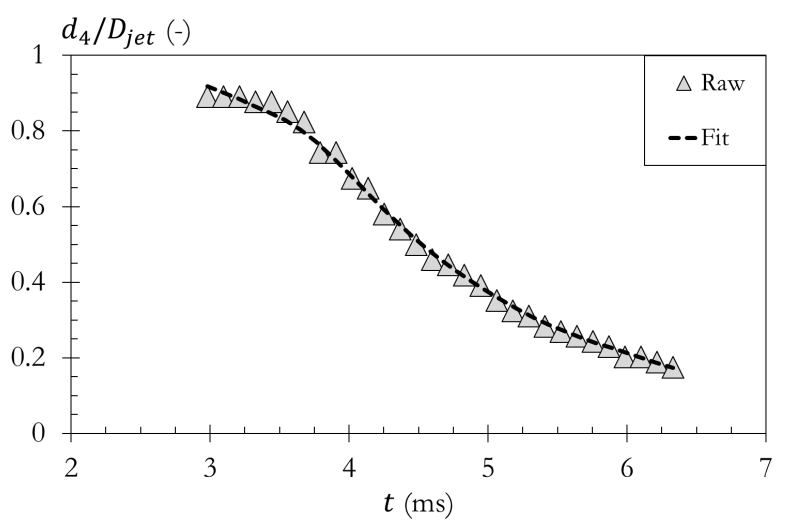

Figure 3. Temporal evolution of the dimensionless scale $d_{4} / D_{\text {jet }}$ (where $D_{\text {jet }}$ is the jet diameter) and mathematical fit. (5 ppm, B3, $Q_{v}=23.710^{-8} \mathrm{~m}^{3} / \mathrm{s}$ )

$$
\eta_{E, a p p}(t)=\frac{-\sigma}{\dot{D}(t)}=\frac{-\sigma}{\dot{d}_{4}(t)}
$$

According to $[4,9]$, the thinning dynamic of the ligament diameter scales as $D(t) / D(0)=$ $\exp \left(-t /\left(3 t_{r}\right)\right)$ during the elasto-capillary regime. However, a recent investigation due to Mathues et al. [6] demonstrated that, because of constant axial tension in the jet arising from the constant creation rate of new surface at the nozzle, a faster exponential thinning is observed. The elasto-capillary regime is replaced by an axial-elastic regime that scales as $D(t) / D(0)=\exp \left(-t /\left(2 t_{r}\right)\right)$. In the present analysis, we decided to apply Mathues et al. [6] scenario and opted for the axial-elastic regime. The dynamics of this regime together with Eq. 2 leads to:

$$
t_{r}=\frac{1}{\dot{\varepsilon}}
$$

Thus, the protocol to determine the relaxation time $t_{r}$ consists in, first, smoothing the experimental $d_{4}(t)$, second, calculating the deformation rate $\dot{\varepsilon}(t)$ as a function of time with Eq. 2, third, identify the value of $\dot{\varepsilon}$ for which a plateau is reached, fourth, use Eq. 4 to determine $t_{r}$. This protocol is illustrated in Fig. 4.

The visco-capillary regime is reached when the polymers have been fully unfolded. The solution has a constant elongational viscosity and behaves as a Newtonian liquid. In this regime, the ligament contraction due to the capillary forces is controlled by the viscous effects and the ligament diameter follows a linear thinning dynamics and scales as $D(t)-D(0)=-\sigma t / \eta_{E, t}$ where $\eta_{E, t}$ is the terminal extensional viscosity of the solution. For such dynamics, it has been 


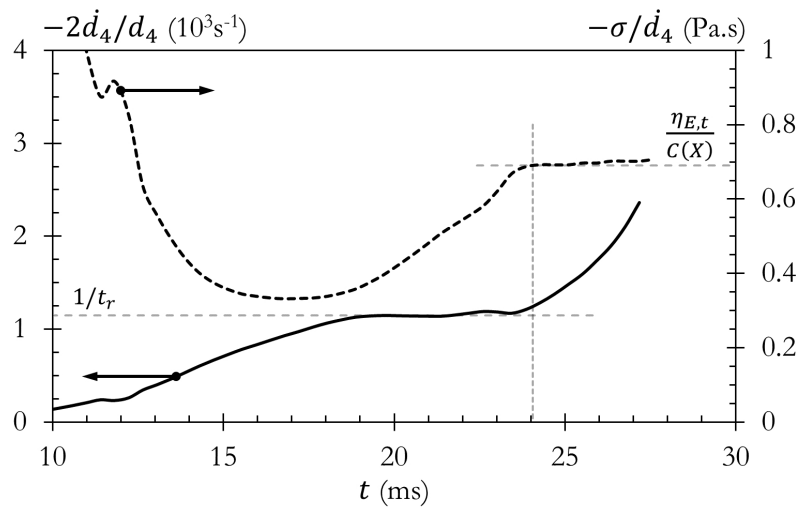

Figure 4. Ligament elongation rate $\dot{\varepsilon}$ (left axis) and apparent extensional viscosity $\eta_{E, a p p}$ (right axis) as functions of time (Solution 5ppm, nozzle B5, $Q_{v}=45.210^{-8} \mathrm{~m}^{3} / \mathrm{s}$ )

demonstrated that the terminal extensional viscosity depends on the temporal derivative of scale $d_{4}(t)$ as [12]:

$$
\eta_{E, t}=\frac{-\sigma C(X)}{\dot{d}_{4}(t)}
$$

In this equation, the parameter $X$ is a measure of the span of the diameter distribution of the ligaments seen at a given position, and $C(X)$ is a correcting coefficient ranging from 0.75 to 3 . When $X=0, C(X)=1$ and $C(X)$ tends towards 3 when $X$ increases to infinity. From these elements, $\eta_{E, t}$ can be estimated by, first, plotting $-\sigma / \dot{d}_{4}(t)$ as a function of time, and, second, identifying the plateau at higher times whose height is equal to $\eta_{E, t} / C(X)$. This procedure is illustrated in Fig. 4.

\section{Results and discussion}

The results are presented in two series. Series 1 gathers a high number of working conditions, including various nozzle diameters and lengths, flow rates and solution concentrations, performed with the polymer with the highest molar weight $\left(M_{w}=810^{3} \mathrm{~kg} / \mathrm{mol}\right)$. The relaxation times for this series are plotted in Fig. 5.

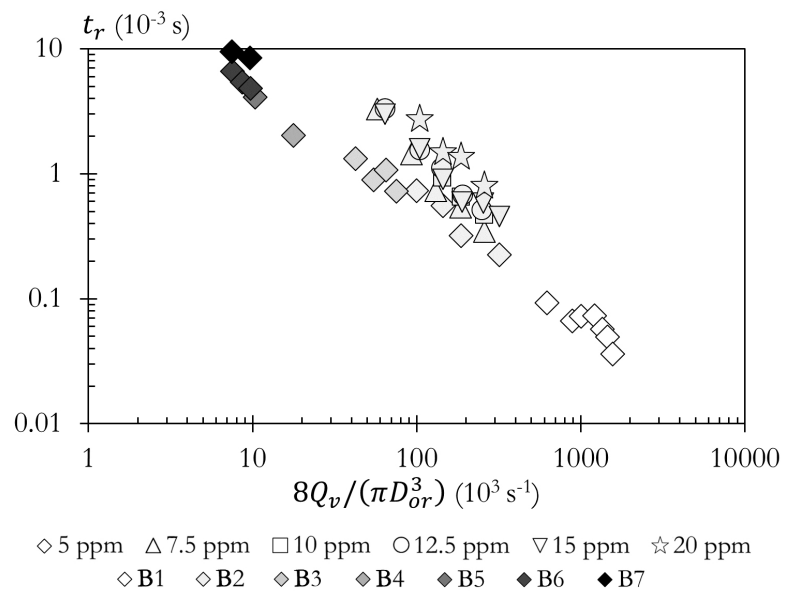

Figure 5. Relaxation time $t_{r}$ as function of the nozzle deformation rate $8 Q_{v} /\left(\pi D_{o r}^{3}\right)$ (Series 1 )

We note that $t_{r}$ depends on the solution concentration, the nozzle diameter and the flow rate. The dependence with the concentration was expected and corresponds to the one reported in the literature ([6] for instance): $t_{r}$ increases with the solution concentration. However, the unexpected influence of the nozzle diameter and the flow rate on $t_{r}$ indicates that the injection 
imposes a mechanism that modifies the physical properties of the solutions. Using the abscissa $8 Q_{v} /\left(\pi D_{o r}^{3}\right)$ (which is equal to $2 V_{o r} / D_{o r}$ and is therefore an indicator of the deformation rate in the injector) in Fig. 5, the results are organized into correlations that depend only on the solution concentration. Thus, the influence of the injection on the relaxation time is related to the deformation rate in the injector independently of the injector diameter or flow rate. The most relevant explanation for this observation is to invoke the presence of mechanical degradation of polymers in the injector. This mechanism is characterized by the rupture of the polymers when they are subjected to sufficiently large deformation rates. Mechanical degradation changes both the mean molecular weight and the molecular weight distribution of the polymers [11]. As a consequence, the relaxation time is also affected by this mechanism. In agreement with other experimental results, we find that the polymer degradation mechanism decreases the relaxation time of the solution.

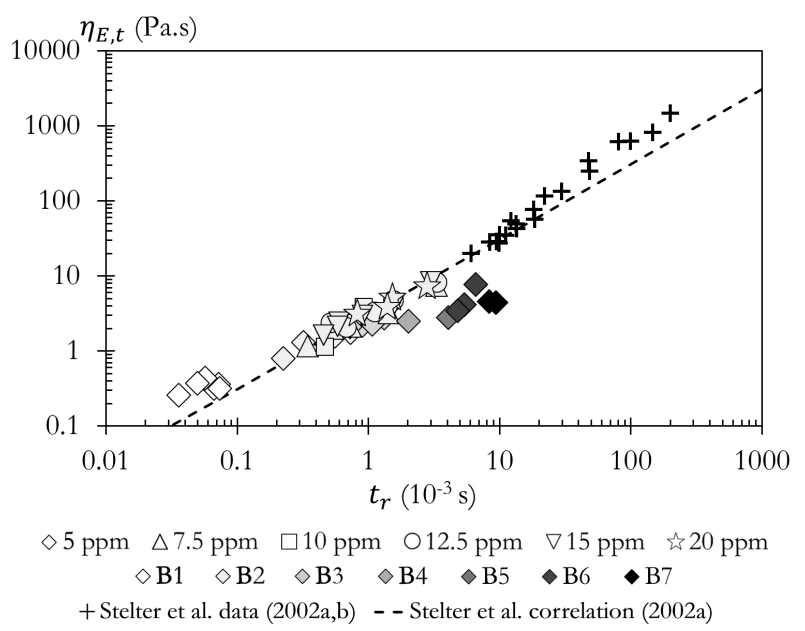

Figure 6. Terminal extensional viscosity $\eta_{E, t}$ as function of relaxation time $t_{r}$ (Series 1). Comparison with Stelter et al. experimental results $[10,11]$ and Stelter et al. model [10]: $\eta_{E, t}=3074.9 t_{r}+0.003$.

The terminal elongational viscosities $\eta_{E, t}$ of the series 1 operating conditions are plotted in Fig. 6 as a function of the relaxation time. Determined with Eq. 5, the elongational viscosities were calculated by considering the coefficient $C(X)$ equal to 1 . This choice results from a study of the ligament diameter distributions observed at a given position [12]. We see in this figure that all experimental results present a nice correlation. Furthermore, this correlation aligns with the experimental results obtained by Stelter et al. $[10,11]$ and therefore extend the validity of their model $\left(\eta_{E, t}=3074.9 t_{r}+0.003\right)$ in the small relaxation time domain.

The second series of measurements (Series 2) concentrated on the influence of the polymer molar weight. A single injector nozzle was used (Nozzle B3, see Table 1) and the concentration was fixed at $5 \mathrm{ppm}$. The relaxation time and terminal elongational viscosity were determined for each solution as a function of the volume flow rate.

The first results to be mentioned concern the solutions with the lightest polymers $(0.6$ and 1 $10^{3} \mathrm{~kg} / \mathrm{mol}$ ) for which, in the tested volume flow rate interval, no elasto-capillary regime could be identified. For these cases, the ligament deformation rates $\dot{\varepsilon}(t)$ were very low and the identification of a plateau was never satisfactory enough to continue the analysis at its completion. It should be noted that the use of very dilute solutions of light and possibly mechanically degraded polymers reduces the elastic properties of the solutions.

For the two other polymers (5 and $810^{3} \mathrm{~kg} / \mathrm{mol}$ ) the relaxation times are plotted as a function of the injector deformation rate $8 Q_{v} /\left(\pi D_{o r}^{3}\right)$ in Fig. 7. For a comparison purpose, the results of series 1 obtained for the $5 \mathrm{ppm}$ concentration are reproduced also in this figure. The series 2 results also report a decrease of $t_{r}$ due to polymer mechanical degradation. We see that the results obtained with the heaviest polymer $\left(810^{3} \mathrm{~kg} / \mathrm{mol}\right)$ are very close to those from series 1 , which is meliorative for the measurement protocol. The results also show that the effect of mechanical degradation on the relaxation time is less pronounced when the polymer molar 
weight decreases.

Finally, the terminal elongation viscosity for the series 2 again reports a correlation with the relaxation time well represented by Stelter et al's model [10]. This is illustrated in Fig. 8.

It can be therefore assumed that, as a first approach. Stelter et al's model can be applied to evaluate either $t_{r}$ or $\eta_{E, t}$. This possibility has been tested here for the lightest polymer solutions. While for these solutions no plateau in deformation rate was identified, the apparent elongational viscosity does converge to a constant value from which a relaxation time can be estimated using Stelter et al's model. Only the polymer solution of molar mass equal to $1.10^{8} \mathrm{~kg} / \mathrm{mol}$ returned acceptable results. Presented in Figs. 7 and 8 , they demonstrate the existence of a mechanical degradation mechanism of the polymers and confirm the sensitivity of this mechanism to the molar mass of the polymer.

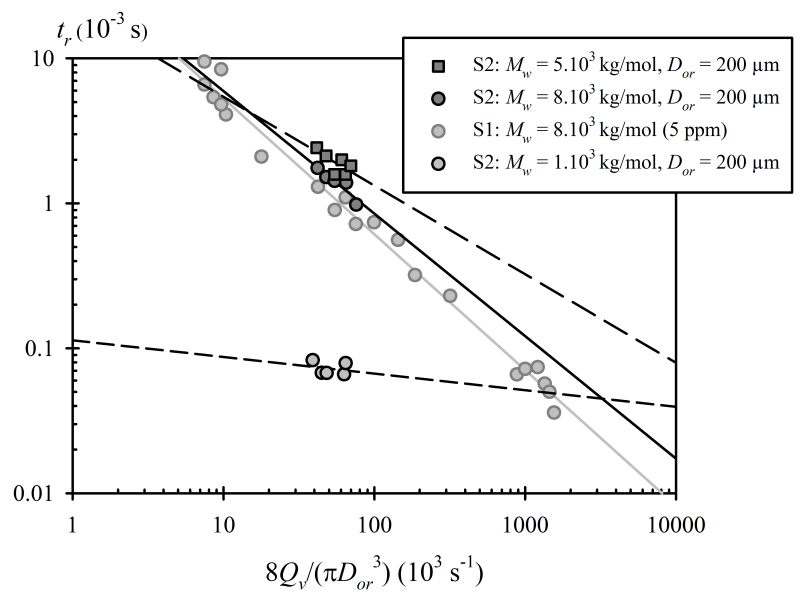

Figure 7. Relaxation time $t_{r}$ as function of the nozzle deformation rate $8 Q_{v} /\left(\pi D_{o r}^{3}\right)$ (Series 2, Comparison with series 1-5 ppm)

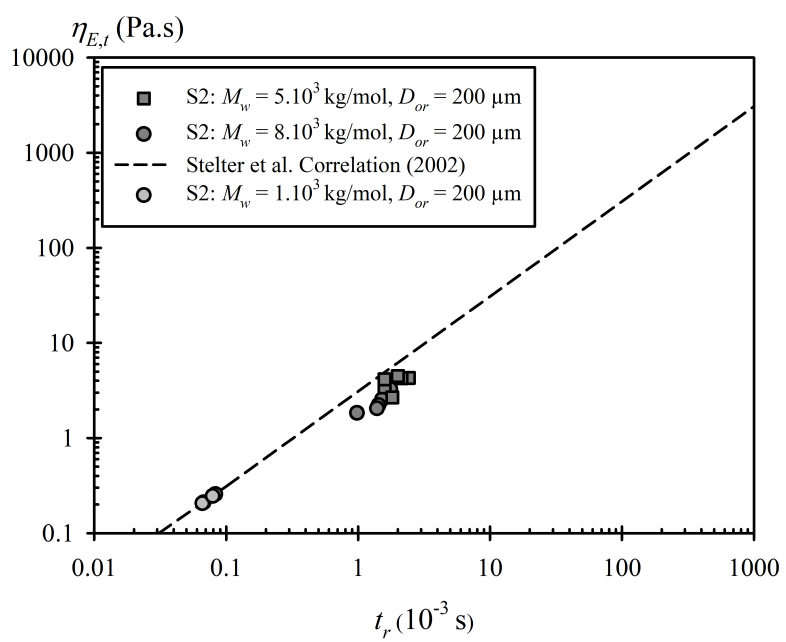

Figure 8. Terminal extensional viscosity $\eta_{E, t}$ as function of relaxation time $t_{r}$ (Series 2). Comparison with Stelter et al. correlation [10]: $\eta_{E, t}=3074.9 t_{r}+0.003$.

\section{Conclusions}

The experimental study presented in this paper demonstrates that the jet rheometer technique can be used to determine the relaxation time and the terminal elongational viscosity of lowviscous visco-elastic solutions. Resulting from a statistical analysis, the solution characteristics are those of the average behaviors. Obtained for a wide range of operating conditions, the results are in good agreement with the model of Stelter et al., although established for a very 
different range of solutions. The results point out the presence of mechanical degradation of the polymers in the injector whose effect is to reduce both the relaxation time and the terminal elongational viscosity of the solution. The polymer degradation mechanism increases with the flow rate following a variation rate that depends on the polymer molar mass: The rate increases as the molar mass increases. This confirms that long polymers are more subjected to degradation than short ones.

The multi-scale analysis proposed here is perfectly suited for the statistic approach imposed by the choice of free injection and its reproducibility is shown in this work. The determination of characteristics such as relaxation time and terminal elongational viscosity is an example of what this analysis can produce, but it contains a lot of other information not yet analyzed. For example, the local distribution of ligament diameters can be obtained and its temporal evolution can inform us about possible heterogeneous behavior.

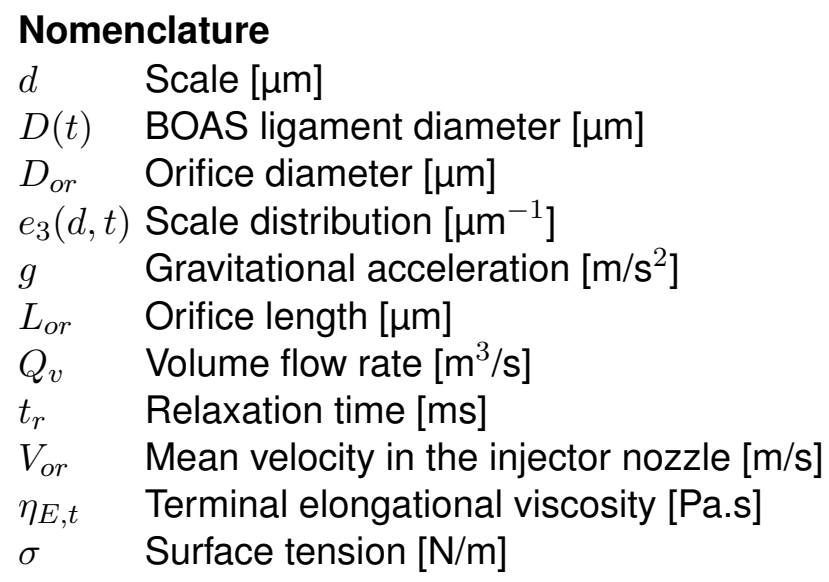

\section{References}

[1] Clasen, C., Phillips P.M., Palangetic, L., Vernant, J., 2012, AIChE J, 58(10) pp. 3242-3255.

[2] Dumouchel, C., July. 30.-Aug. 3. 2017, ASME-FEDSM, FEDSM2017-69590.

[3] Dumouchel, C., Thiesset, F., Ménard, T., Aug. 29.-Sept. 2. 2021, ICLASS 2021.

[4] Entov, V.M., Hinch, E.J.,1997, J. Non-Newtonian Fluid Mech., 72(1) pp. 31-53.

[5] Keshavarz, B., Sharma, V., Houze, E.C., Koerner, M.R., Moore, J.R., Cotts, P.M., ThrelfallHolmes, P., McKinley, G.H.,2015, J. Non-Newtonian Fluid Mech. 222, pp. 171-189.

[6] Mathues, W., Formenti, S., Mcllroy, C., Harlen, O.G., Clasen, C., 2018, Journal of Rheology, 62(5) pp. 1135-1153.

[7] McKinley, G.H., 2005,In: Binding DM, Walter K (ed) Rheology annual. The Royal Society of Rheology, Edinburgh, pp. 1-49.

[8] Schümmer, P., Tebel, K.H., 1983, J. Non-Newtonian Fluid Mech., 12(3) pp. 331-347.

[9] Stelter, M., Brenn, G., Yarin, A.L., Singh, R.P., Durst, F., 2000 , Journal of Rheology, 44(3), pp. 595-616.

[10] Stelter, M., Brenn, G., Durst, F., 2002, Atomization and Sprays, 12, pp. 299-327.

[11] Stelter, M., Brenn, G., Yarin, A.L., Singh, R.P., Durst, F., 2002, Journal of Rheology, 46, pp. 507-527.

[12] Tirel, C., Renoult, M.C., Dumouchel, C., 2020, Experiments in fluids, 61, n²1, pp. 1-14. 